\title{
COARSE PARTICLES' THRESHOLD OF MOTION UNDER SHOALING WAVES
}

\author{
Marcel J.F.Stive ${ }^{1}$, Ad J.H.M. Reniers ${ }^{1}$, Emanuele Terrile ${ }^{2}$, Henk Jan Verhagen ${ }^{1}$ \\ ${ }^{1}$ Faculty of Civil Engineering and Geosciences, Delft University of Technology, PO \\ Box 5048, 2600 GA Delft, The Netherlands, M.J.F.Stive@citg.tudelft.nl, \\ A.J.H.M.Reniers@citg.tudelft.nl, H.J.Verhagen@citg.tudelft.nl \\ ${ }^{2}$ D.I.Am., University of Genoa, Italy, Terrile@diam.unige.it
}

\begin{abstract}
The threshold of motion of coarse particles under regular shoaling waves is studied. Experiments are performed to investigate the effects of bed fluid acceleration on coarse particle stability. By varying wave height, wave period and water depth, combinations of similar peak orbital velocities and weak to strong intra-wave accelerations were created. Qualitatively we found that initiation of motion occurs at or is very close to the maximum shear stress due to the combined effects of drag/lift and acceleration as introduced by Nielsen and Callaghan (2003). However, quantitatively their formulation does not lead to convincing discrimination between motion and no motion. We expect this to be due to the assumption that the coefficients for drag/lift and acceleration in their formulation are taken equal and constant. From literature and from plotting our data against the Keulegan-Carpenter number we expect that these coefficients strongly vary due to flow separation effects.
\end{abstract}

\section{INTRODUCTION}

Coastal sediment transport researchers have been aware of the importance of fluid accelerations or the associated horizontal pressure gradients for a long time. Bagnold (1963) already recognised the potential importance of wave-induced accelerations in general. Madsen (1974) showed that horizontal pressure gradients associated with steep fronts of waves or bores might induce bulk instability and hence vastly enhanced shoreward sediment transport. Nielsen (1979) discussed the likely 
Keulegan-Carpenter number effects associated with accelerations in wave sediment transport. An interesting experiment was done by King (1991) measuring different net sediment transport rates for forward facing and backward facing saw-tooth halfwaves. The importance of surf zone waves often having saw-tooth asymmetry was discussed by Nielsen (1992), which brings this author to the conclusion that it leads to acceleration asymmetry and to thinner boundary layers ( greater shear stresses) associated with those peak velocities, which follow the briefest acceleration process. Sleath (1994) defined a quasi-steady regime and a pressure gradient-regime for coastal sediment transport separated by the value an acceleration parameter, and in several subsequent works, e g, Zala-Flores and Sleath (1998) and Sleath (1999) acceleration effects in wave induced sediment transport were quantified in terms of this parameter. The sheetflow data of Ribberink et al (2000) showed that real waves in a flume, as opposed to Stokes-wave like velocities in U-tubes generated at least two times more sediment transport for the same orbital velocity magnitude, a difference which could be due to either saw tooth asymmetry or boundary layer streaming being present in the flume waves but not in the U-tube experiments. The discussion of Nielsen \& Callaghan (2003) subsequently provided quantitative estimates of the relative importance of streaming versus acceleration asymmetry. Drake and Calantoni (2001) made a quantitative process-based model to incorporate the effect of pressure gradients on particle motion. Hoefel and Elgar (2003) used their results to show that flow acceleration may play a role in predicting onshore bar movement under moderate wave conditions. Although the effect of flow acceleration for fine particles is not well established yet, the above findings inspired our research group to undertake a series of experiments to further explore the role of flow acceleration. Since the effect is stronger for coarser particles (larger spatial pressure gradients) we decided to undertake experiments for unsteady flow with coarse particles of $(\mathrm{O}) \mathrm{cm}$ diameter. In the region just before wave breaking near bed fluid accelerations are found to be strong enough to move the coarse sediment. While the relevance for sand transport has yet to be established, the relevance for stability of bed protection and for onshore gravel transport clearly exists.

\section{EXPERIMENTAL SET-UP AND METHOD}

The experiments were carried out in a wave flume of the Laboratory of Fluid Mechanics of Delft University of Technology. The glass-walled flume has an effective length of $42.00 \mathrm{~m}$, a width of $0.80 \mathrm{~m}$ and a height of $1.00 \mathrm{~m}$. On the bottom of the flume a concrete slope was constructed with a gradient of 1:30 (Figure 1). Regular waves were generated with a wave paddle using second order wave steering and measurements were confined to the shoaling region. In order to compensate the influence of re-reflecting waves in the flume, an Active Reflection Compensation system was used. Surface elevations were measured with six resistance type wave gauges (Figure 1). Measurements confirmed that reflection coefficients on the 1 in 30 slope were low, viz. $1,2 \%$ on average.

Fluid motions were measured with an EMS (Electro Magnetic Flow Sensor) positioned about $5 \mathrm{~cm}$ above the bottom at various locations along the slope. Intra- 
wave variation of the orbital velocities and accelerations were derived by ensemble averaging over the wave phase (records between 30-50 seconds were used implying averaging over 10 to 16 waves).

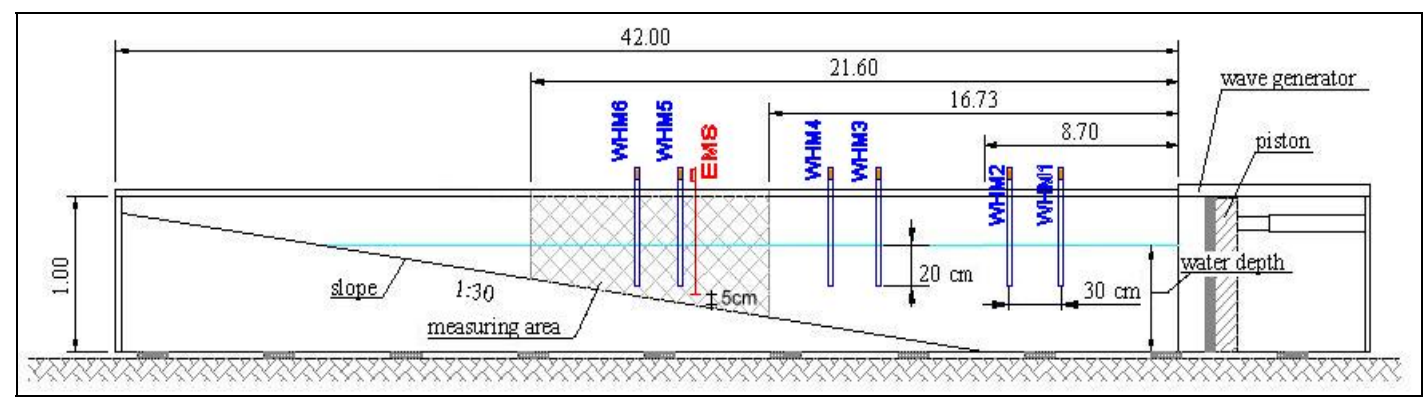

Figure 1: Flume dimensions and location of measurement instruments along the slope (horizontal dimensions in meters)

Two types of nearly uniform coarse particles $\left(\mathrm{D}_{90} / \mathrm{D}_{10} \cong 1.25\right)$ were applied in the experiments: $\mathrm{D}_{50}=8.8 \mathrm{~mm}$ and $\mathrm{D}_{50}=11.4 \mathrm{~mm}$. The specific density of the particles was $2.67 \mathrm{~kg} / \mathrm{m}^{3}$. In the shoaling region 5 strips of coloured particles (different colours per strip) were placed that could move, while outside these strips the particles were fixed to the bottom. For each test performed the number of particles were determined that moved by observing the measuring area at a particular strip, and the associated forcing wave flow properties, viz. the intra-wave near-bed velocity and acceleration variations. The tests were carried out with different wave periods in the range between $\mathrm{T}=2 \mathrm{~s}$ and $\mathrm{T}=4.4 \mathrm{~s}$, with different wave heights: 0.125 , $0.15,0.175,0.20 \mathrm{~m}$. and at two water depths $\mathrm{h}=0.60$ and $0.65 \mathrm{~m}$ respectively.

In order to create a statistically significant dataset a sufficient number of forcing situations is needed to establish the threshold of coarse particle motion, i.e. we need a sufficient number of situations with similar orbital velocity magnitudes in combination with small to large acceleration magnitudes. Some of these combinations did not lead to particle movement while others with similar orbital velocities, but larger acceleration did lead to particle movement. To establish a reliable percentage of particle motion, tests with near-equal velocities and accelerations for which particle motion occurred were repeated multiple times (up to 6 or 7 times). In doing so we were able to reduce stochastic effects, for instance due to insufficient settling time of the bed after placing which can result in a motionfavourable position for a particle. A total of 122 experimental results were established covering a range of orbital velocities and orbital accelerations.

As indicated the stochastic nature of the particle movement needs consideration, therefore we have categorized the particle movement in classes (Table 1). Threshold of motion is defined as movement of at least 3 coloured particles out of a coloured strip. Movement of particles within a strip is not considered as movement. The percentages refer to the ratio of the times that three stones moved over the total of times that particle movement was assessed under near-equal conditions. 
Table 1: Classes of stones movement

\begin{tabular}{ll} 
Particle movement percentage & Classification \\
\hline Less than $25 \%$ & Never \\
Between $25 \%$ and $75 \%$ & Sometimes \\
More than $75 \%$ & Always \\
\hline
\end{tabular}

Using movement of at least 3 particles reduces the influence of the orientation of a particle in the bed. A sensitivity analysis, in which the data of the motion of 1 and 4 particles were also analysed, shows small differences with the results found for the chosen criterion of movement of 3 particles.

The above approach results in a dataset, which determines no, some, or significant particle movement with associated peak orbital motion and a representative measure of acceleration. This dataset forms the basis of our analysis presented below.

\section{DATA ANALYSIS}

As a first step in our data analysis we discuss our visual and video observations. Figure 2 depicts a typical time evolution of the free stream orbital velocity and the associated accelerations. Initiation of motion was observed to occur consistently in between the moment of maximum acceleration (point B) and the moment of maximum onshore flow velocity (point C). Apparently, the combination in this region of high acceleration and high free stream velocity creates an optimum condition for sediment particle instability.

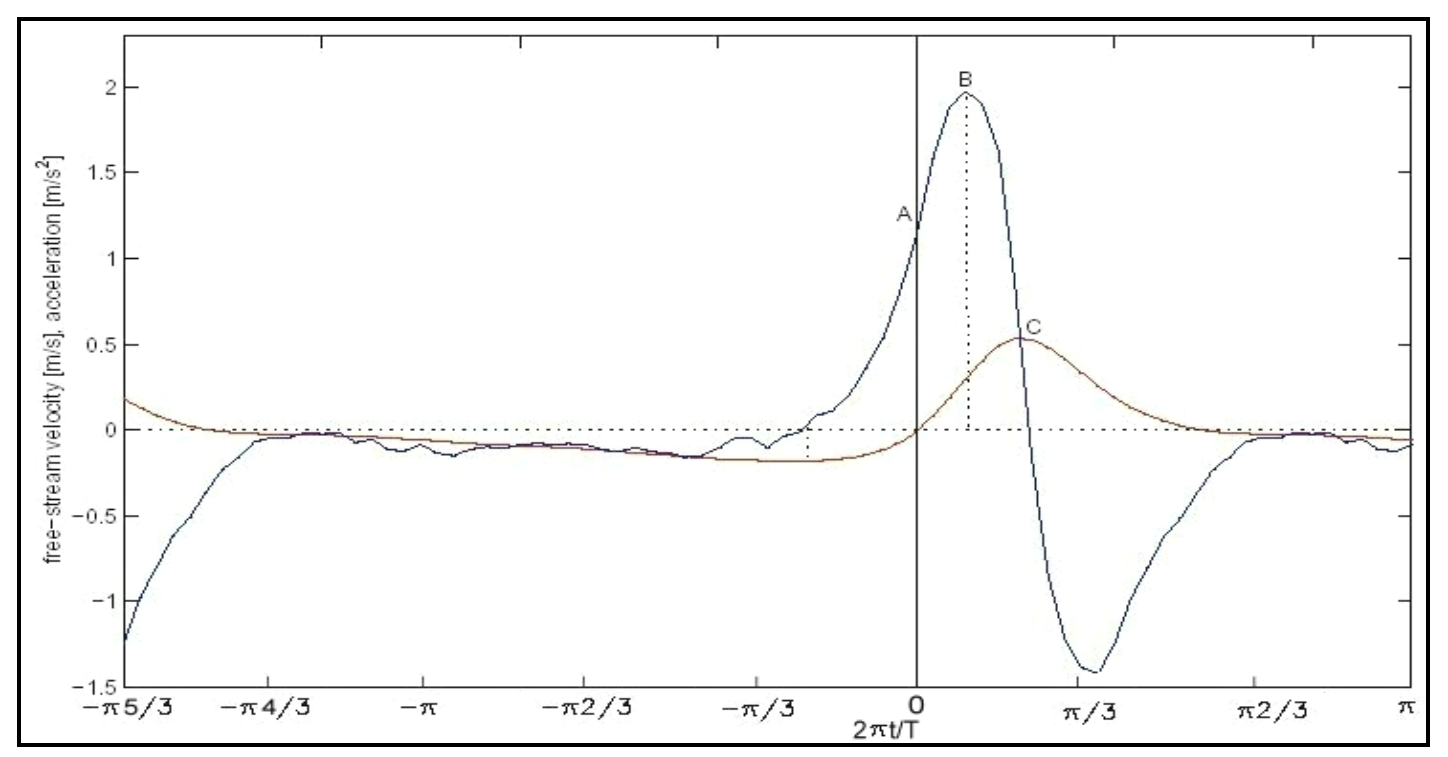

Figure 2: Time series of near-bed velocity (red line) and acceleration (green line). 
The above analysis can be made quantitative by making use of the formulation proposed by Nielsen $(1992,2002)$ and Nielsen and Callaghan (2003) for the Shields parameter in an unsteady turbulent flow:

$$
\theta_{a}(t)=\frac{\frac{1}{2} f_{2.5}}{(s-1) g D_{50}}\left(u_{\infty} \cos \varphi_{\tau}+\frac{1}{\omega_{p}} \sin \varphi_{\tau} \frac{d u_{\infty}}{d t}\right)^{2} \times \operatorname{sign}\left(u_{*}(t)\right)
$$

where $f_{2.5}$ is the wave friction factor (Jonsson, 1966 and Jonsson and Svendsen, 1976) corresponding to a bed roughness of $2.5 D_{50}$ (Nielsen, 1992), $s$ is the relative density $\left(\rho_{s} / \rho\right), u_{\infty}$ is the free-stream velocity, $\omega_{p}$ is the peak angular frequency $(2 \pi / T)$ and $\varphi_{\tau}$ is the phase shift between free stream velocity and bed shear stress at the peak frequency. Note that in contrast to Nielsen (1992) the square of the summed effect instead of a linear summation of shear velocity and acceleration is taken in order to get the correct representation of the sediment flux bursts in a turbulent flow. Also note that for both effects the same coefficient is used, which will be discussed below.

Equation (1) expresses both the effect of shear velocity (first term) and of acceleration (second term) on the stability parameter $\theta_{a}$. Making use of our measurements and by adopting a value of $1 / 18 \pi$ for $\varphi_{\tau}$ (which we have quantified from video observations and which is in line with Fredsoe and Deigaard, 1992) we have computed a typical intra-wave variation of the three contributing terms (Figure $3)$.

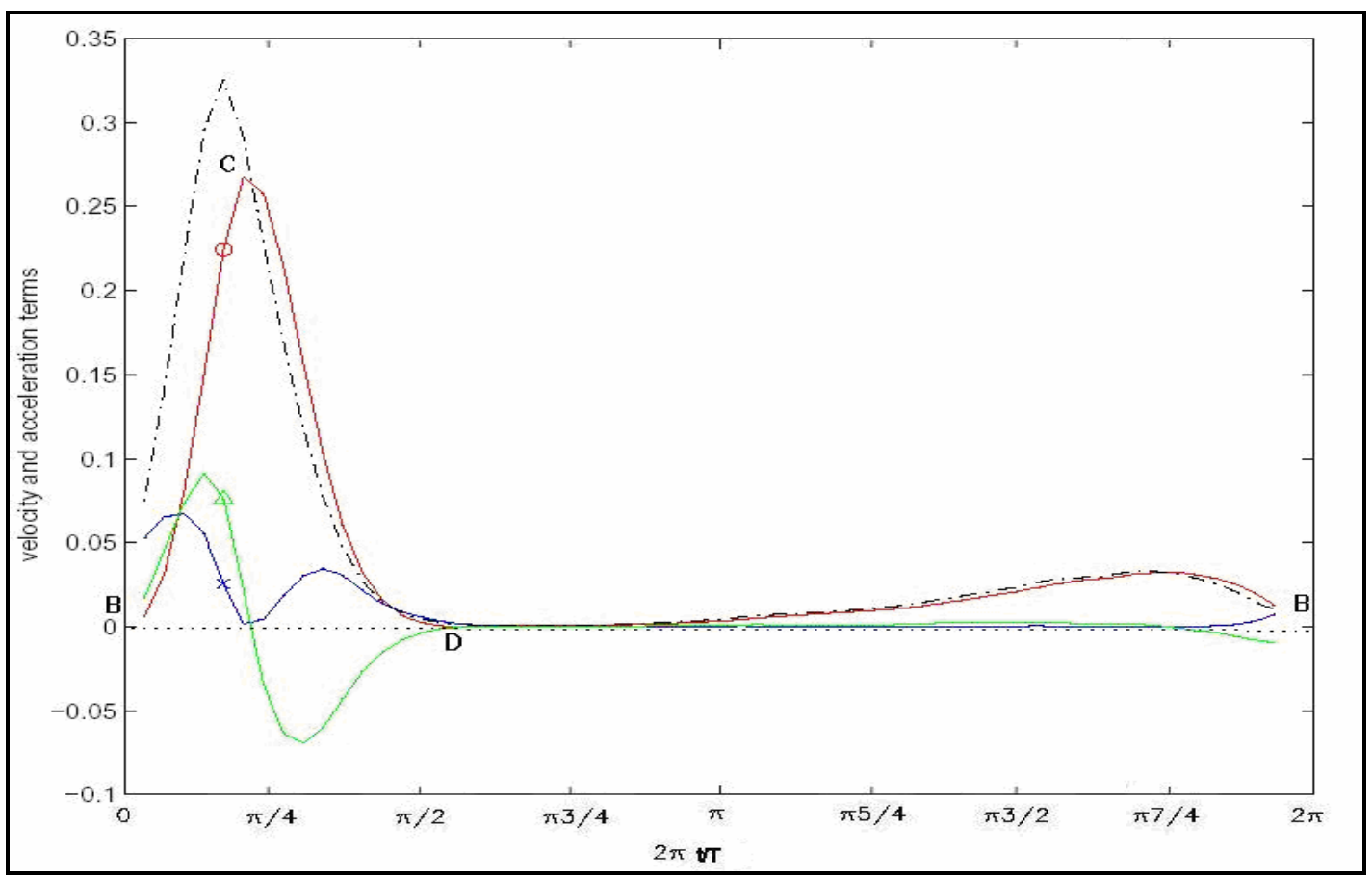

Figure 3: Velocity and acceleration terms of the stability parameter and the stability parameter $\theta_{a}$ for a wave with period $\mathrm{T}=3 \mathrm{~s}$ and height $\mathrm{H}=0.15 \mathrm{~m}$ (see text for explanation). 
The solid line, containing the symbol o, represents the pure velocity contribution $\left(u_{\infty} \cos \varphi_{\tau}\right)^{2}$, the solid line, containing the symbol $\mathrm{x}$, represents the acceleration contribution $\left(1 / \omega_{p} \sin \varphi_{\tau} \mathrm{d} u_{\infty} / \mathrm{d} t\right)^{2}$, the solid line, containing the symbol $\Delta$, represents their cross-product $\left(u_{\infty} \cos \varphi_{\tau} 1 / \omega_{p} \sin \varphi_{\tau} \mathrm{d} u_{\infty} / \mathrm{d} t\right)$ and the dash dot line the total value of the stability parameter $\theta_{a}$.

All three contributions are positive and strong for the particular region between point $\mathrm{B}$ and the passage of the wave crest. The instant at which the sum of these three terms is maximum, corresponding to a maximum value of the Shields' number, is marked by the three symbols $(\Delta),(\mathrm{x})$ and $(\mathrm{o})$. This corresponds to the region between (point $\mathrm{B}$ ) and the maximum onshore free stream velocity (point $\mathrm{C}$ ), and is equal or very close to the instant of motion initiation that we have observed from video.

Qualitatively these results support Nielsen and Callaghan's (2003) formulation as given by Equation (1), i.e. we expect that the maximum value of their formulation should correlate with motion initiation. Following Shields we therefore introduce a particle Reynolds-like number, since it is clear that the maximum orbital velocity and the stone diameter play a role as well:

$$
\operatorname{Re}_{g}=\frac{u_{r m s} D_{50}}{v}
$$

where $v$ is the kinematic viscosity of the fluid and $u_{r m s}=\sqrt{\operatorname{Var}\left[u_{\infty}(t)\right]}$.

Figures 4 and 5 show Nielsen and Callaghan's (2003) formulation for our experiments against the particle Reynolds-like number, where in Figure 5 we have used $u_{\max }$ instead of $u_{\text {rms }}$. As we observe there is a poor discrimination, which is surprising because of the qualitative agreement concluded before.

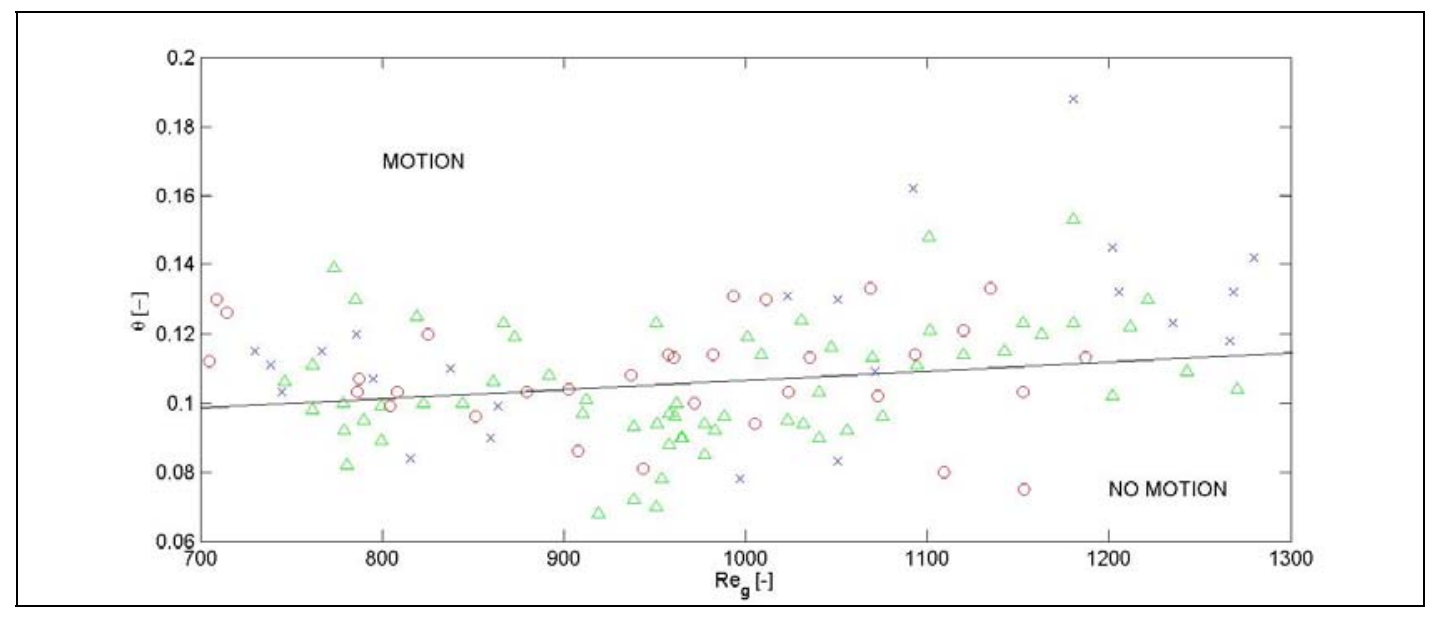

Figure 4: Shields like presentation of Nielsen's maximum shear stress vs Re (based on $\mathrm{u}_{\mathrm{rms}}$ ); triangles, circles and crosses represent no-motion, some-motion and always-motion respectively. 


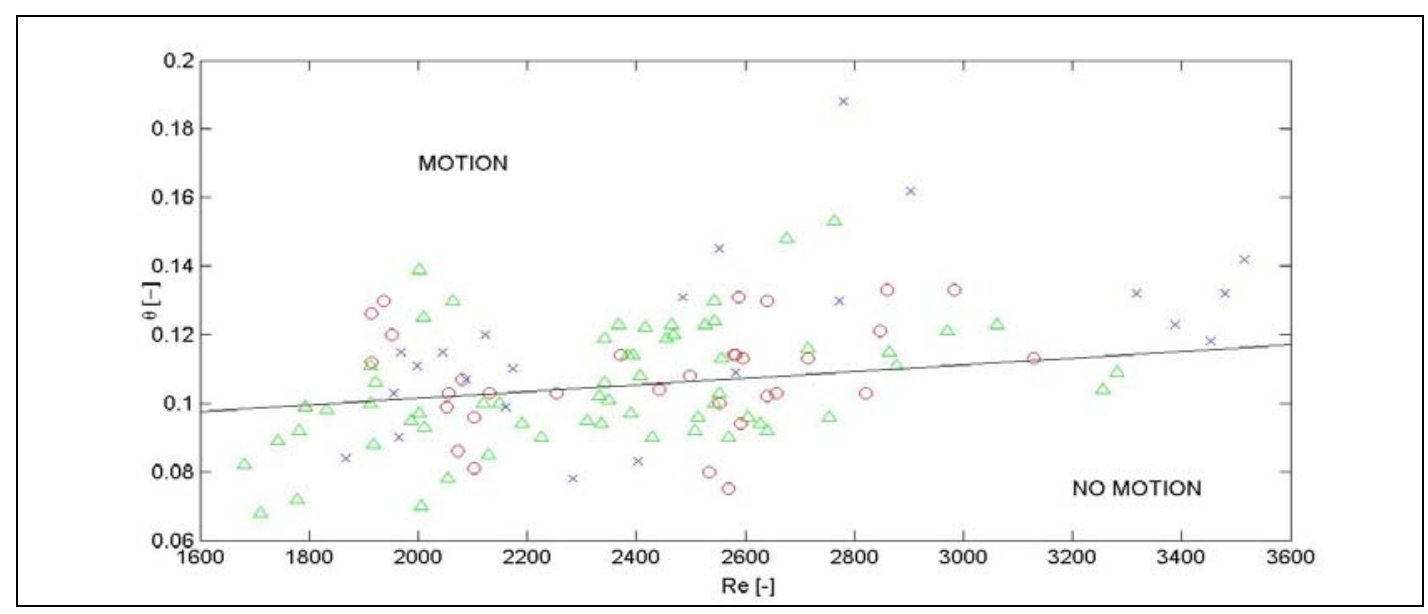

Figure 5: Shields like presentation of Nielsen's maximum shear stress vs Re (based on $u_{\max }$ ); triangles, circles and crosses represent no-motion, some-motion and always-motion respectively.

As a first step in our further exploration of the issue we decided to first look for discrimination between motion and no-motion by presenting our data as a function of a representative acceleration value, since our dataset contains situations with a similar orbital flow velocity and varying acceleration. From the above findings we concluded that two acceleration values play a crucial role in the process of initiation of motion, viz. $a_{0}$ being the value of acceleration at the beginning of the growth of the boundary layer when the velocity is zero and changing to onshore flow (point A in Figure 2) and $a_{\max }$ being the maximum of the near-bed fluid acceleration (point B in Figure 2). Obviously $a_{\max }$ should be large, but when $a_{0}$ is large as well the waves are either very skewed (saw-tooth like) or highly non-linear in a horizontal sense (horizontally asymmetric) and in both cases both the shear velocity and the acceleration are large, the combination of which favours motion. Therefore when the product of these values is large we should expect the conditions most conducive for initiation of sediment particle motion due to the acceleration. We thus introduce a new dimensional acceleration descriptor for nonlinear waves defined as follows:

$$
a_{n l}=\sqrt{a_{\max } a_{0}}
$$

The following properties for $a_{n l}$ are noted. Its value always lies between the value of $a_{0}$ and $a_{\max }$; in case of skewed waves, the closer it is to $a_{\max }$ the skewer or the more horizontally asymmetric the waves are. For waves that are horizontally asymmetric only, $a_{0}$ and $a_{\max }$ could be either the accelerations at and right after the change to onshore or the accelerations just before and at the change to offshore flow. In both cases the initiation of motion should be similarly likely. For pure sinusoidal waves no difference between $a_{\max }$ and $a_{0}$ exists, hence $a_{\max }$ is $a_{0}$, so $a_{n l}$ equals $a_{0}$, denoting minimal acceleration effects. 
All our experimental results are collected in Figure 6 where for each test the measured $a_{n l}$, made dimensionless by the acceleration of gravity, is plotted versus the particle Reynolds-like number $\mathrm{Re}_{\mathrm{g}}$. A clear division is observed between a region in which there is always movement and one where there is no movement. We have included a threshold of motion region indicative for the separation between motion and no motion. Apparently, for a particular value of $R e_{g}$ we observe a critical value of $a_{n l}$. When $a_{n l} \geq a_{n l, c r i t}$ the particles start to move.

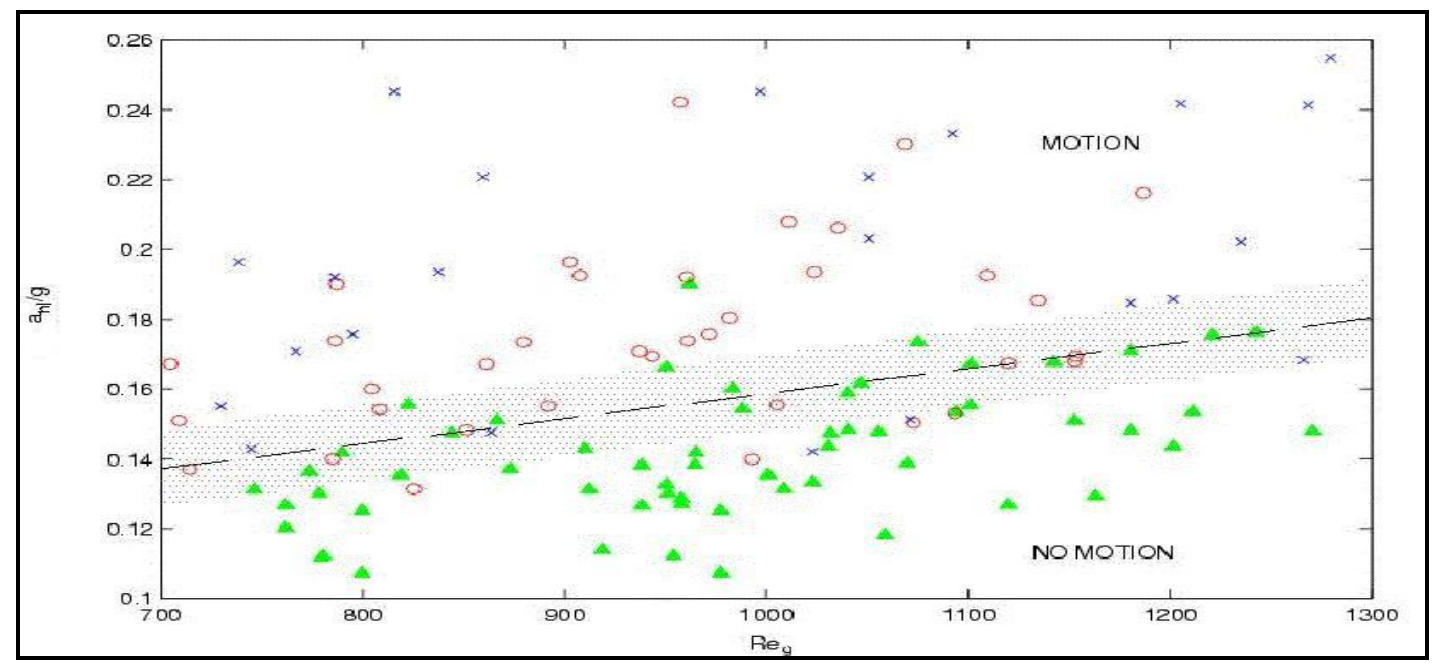

Figure 6: Threshold of motion line $(\Delta)$ never, (o) sometimes, $(x)$ always, as a function of $a_{n} / g$ and $\mathrm{Re}_{\mathrm{g}}$.

Note that this particle Reynolds-like number increases with $D_{50}$ when $u_{r m s}$ is constant. If we expect the acceleration to play a role, initiation of motion should occur for larger values of $a_{n l}$, which is what we observe. On the other hand the number increases with $u_{r m s}$ while $D_{50}$ is constant. Apparently, even when the orbital velocity increases, we need an associated increase of acceleration, such that initiation of motion occurs only for larger values of $a_{n l}$. Initially we found this result difficult to interpret physically. To extend our analysis we chose to plot (see Figure 7) $a_{n l} / g$ versus $u_{r m s}^{2} /\left(g D_{50}\right)$, which represent the two "Morrison-like" theoretical forces (drag/lift and pressure gradient) that cause the motion. We expected a linear decrease of the threshold motion line expressing compensation of the one force by the other, but although the tendency is not as clear as in Figure 6 the initiation of motion line is not decreasing but slightly increasing. Both these results can only be explained when the $C_{d}$ coefficient (the combined drag coefficient) decreases stronger than the $C_{m}$ coefficient (the acceleration coefficient) as $R e_{g}$ increases and a higher acceleration effect is required to compensate for the decrease in shear velocity effect. Looking at the results of Keulegan and Carpenter (1958) and Sarpkaya (1976) this can be observed to be the case for a variety of experiments in ranges of the Reynolds like number similar to our experiments. 


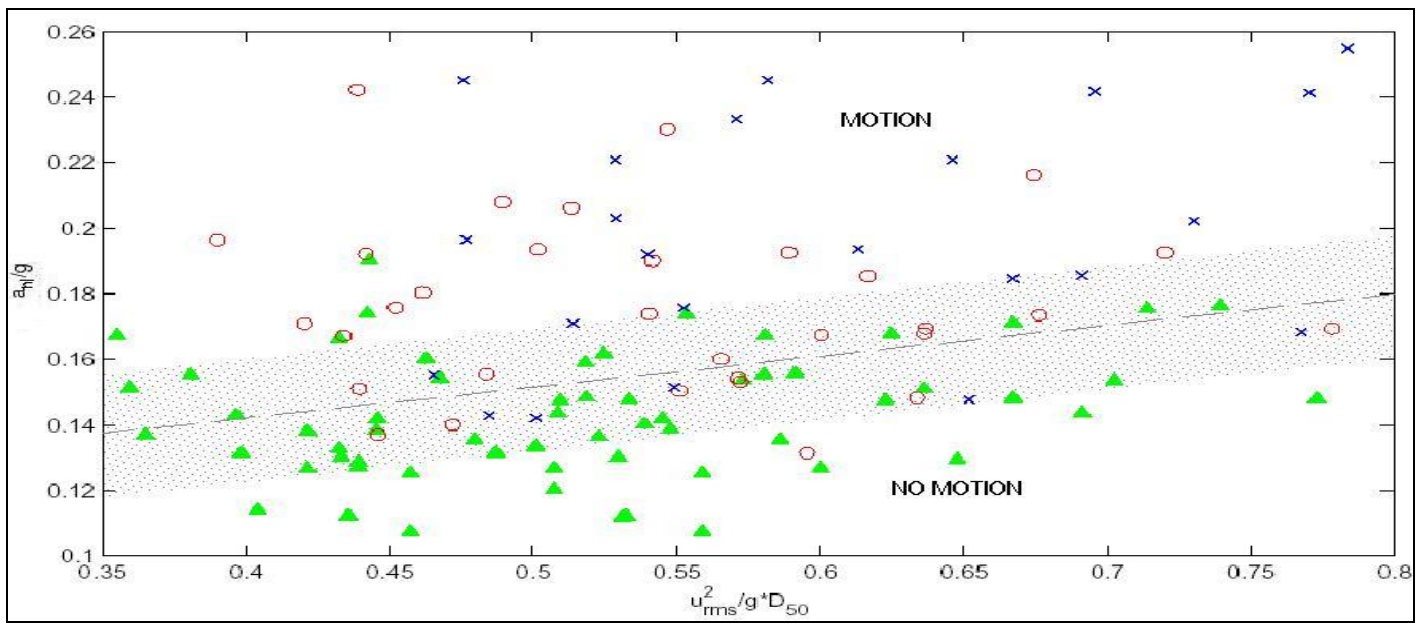

Figure 7: Threshold of motion line ( $\Delta$ ) never, (o) sometimes, $(x)$ always, as a function of $a_{n} / g$ and $u^{2}{ }_{r m s} / g D_{50}$.

These conclusions are confirmed by plotting our results against the KeuleganCarpenter number in Figure 8, which indicates that apparently our results fall in the region where strong variations are encountered in the degree of flow separation which influences both drag and acceleration. This conclusion makes the use of the same coefficient for the drag/lift and acceleration effect in Equation (1) a questionable assumption for the present dataset.

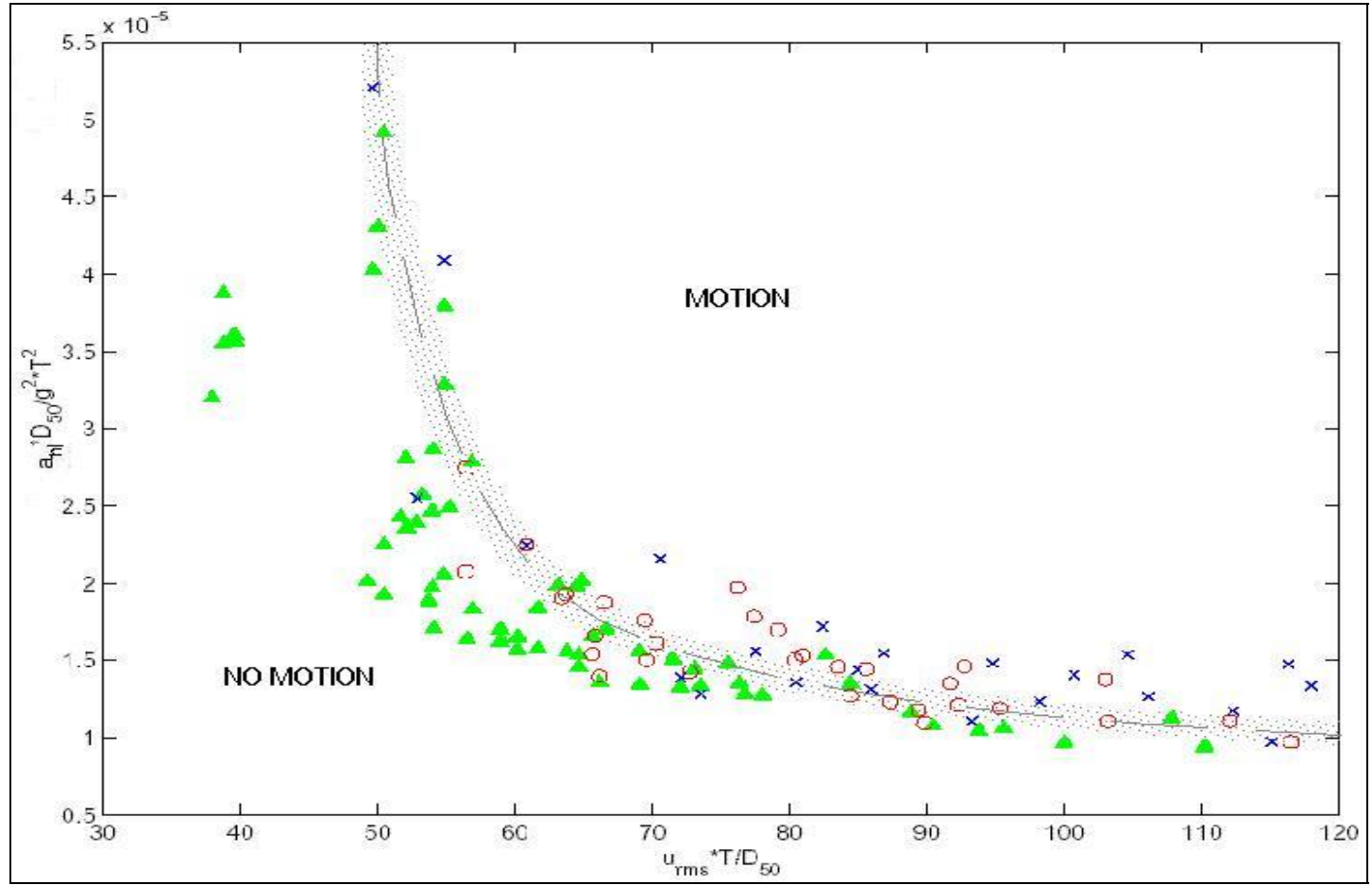

Figure 8: Threshold of motion line ( $\Delta$ ) never, (o) sometimes, $(x)$ always, as a function of $a_{n 1}$ $D_{50} / g^{2} T^{2}$ and the Keulegan-Carpenter number $u_{r m s} T / D_{50}$. 


\section{DISCUSSION AND CONCLUSION}

Based on experiments with regular shoaling waves the effects of bed fluid acceleration on coarse particle stability were investigated for two different sizes, both of a $\mathrm{cm}$ order-of-magnitude. By varying wave height, wave period and water depth combinations of similar peak orbital velocities and weak to strong intra-wave accelerations were created. The data confirmed that the acceleration plays a role for the initiation of motion, since combinations of similar orbital velocity and varying acceleration magnitude resulted in no motion, some motion and motion as acceleration increased.

Qualitatively we found that initiation of motion occurs at or is very close to the maximum shear stress due to the combined effects of drag/lift and acceleration as introduced by Nielsen and Callaghan (2003). However, quantitatively their formulation does not lead to convincing discrimination between motion and no motion. We expect this to be due to the assumption that the coefficients for drag/lift and acceleration in their formulation are taken equal and constant. From literature and from plotting our data against the Keulegan-Carpenter number we expect that the coefficients strongly vary caused by flow separation effects.

\section{ACKNOWLEDGEMENTS}

The experiments were conducted in a wave flume of the Hydraulics Laboratory of the Faculty of Civil Engineering and Geosciences of Delft University of Technology. Maarten Tromp conducted a first set of experiments as part of his MSc Thesis work, which laid the basis for the experiments conducted by Emanuele Terrile as part of his MSc thesis work (supported by the MSc EU Erasmus programme) reported here. Ad Reniers was funded by the Dutch National Science Foundation (NWO), contract DCB 5856. The technical assistance in conducting these experiments by Dr Henry Fontijn is greatly appreciated. The paper improved considerably from discussions with Prof Peter Nielsen.

\section{REFERENCES}

Bagnold, R.A. (1963). "Beach and nearshore processes: Part I, Mechanics of marine sedimentation," In N M Hill Ed, The Sea Vol 3: The Earth beneath the Sea, 507553.

Drake, T.G., and Calantoni, J. (2001). "Discrete particle mode for sheet flow sediment transport in the nearshore," Journal of Geophysiscal Res. Oceans, Vol. 106, No C9, 19.859-19.868.

Elgar, S., and Guza, R.T. (1985). "Observation of bispectra of shoaling surface gravity waves," Journal of Fluid Mechanics, Vol. 167, 425-448.

Fredsøe, J., and Deigaard, R. (1992). "Mechanics of coastal sediment transport," Advanced Series on Ocean Engineering, Vol. 2, World Scientific. 
Hoefel, F., and Elgar, S. (2003). "Wave-induced sediment transport and sand bar migration," Science, Vol. 299, 1885-1887.

Jonsson, I.G. (1966). "Wave boundary Layers and friction factor," Proceedings $10^{\text {th }}$ Int Conf Coastal Eng, Tokyo, ASCE, New York.

Jonsson, I.G., and Svendsen, I.A. (1976), "Hydrodynamics of coastal regions," Technical University of Denmark.

Keulegan, G.H. and Carpenter, L.H. (1958). "Forces on cylinders and plates in an oscillating fluid," J. Res. Nat. Bur. Stand., Vol. 60, No. 5.

King, D.B., Jr (1991). "Studies on oscillatory flow bedload sediment transport," $P h D$ thesis, U C San Diego (Scripps).

Madsen, O.S. (1974). "Stability of a sand bed under breaking waves," Proceedings $14^{\text {th }}$ Int Conf Coastal Eng, Copenhagen, ASCE, 776-794.

Nielsen, P. (1979). "Some basic concepts of wave sediment transport," PhD Thesis, Series Paper 20, ISVA, Tech Univ Denmark.

Nielsen, P. (1992). "Coastal bottom boundary layers and sediment transport," Advanced Series on Ocean Engineering, Vol. 4, World Scientific.

Nielsen, P. (2002). "Shear stress and sediment transport calculations for swash zone modelling," Coastal Engineering, Vol. 45, 53-67.

Nielsen, P., and Callaghan, D.P. (2003). "Shear stress and sediment transport calculations for sheet flow under waves," Coastal Engineering, Vol. 47, 347-354.

Ribberink, J. S., Dohmen-Janssen, C.M., Hanes, D.M., McLean, S.R. and Vincent, C. (2000). "Near bed sand transport mechanisms under waves," Proceedings $21^{\text {th }}$ Int Conf Coastal Eng, Sydney, ASCE, New York, 3263-3276.

Sarpkaya, T. (1976). "Vortex shedding and resistance in harmonic flow about smooth and rough circular cylinders at high Reynolds numbers," Rep. NPS59SL76021, U.S. Naval Postgraduate School.

Sleath, J.F.A. (1994). "Bedload transport in oscillatory flow," In Belogey, Rajaona \& Sleath Ed: Sediment transport mechanisms in coastal environments and rivers (Proc Euromech 310), World Scientific.

Sleath, J.F.A. (1999). "Conditions for plug formation in oscillatory flow," Continental Shelf Res, Vo119, 1643-1664.

Terrile, E. (2004). "The threshold of motion of coarse sediment particles by regular non-breaking waves," MSc Thesis, University of Genoa and Delft University of Technology (www.hydraulicengineering.tudelft.nl).

Tromp, M. (2004). "The influence that fluid accelerations have on threshold of motion," MSc Thesis, Delft University of Technology (www.hydraulicengineering.tudelft.nl).

Zala-Flores, N, and Sleath, J.F.A. (1998). "Mobile layer in oscillatory sheet flow," Journal Geophysical Res., Vol 103, No C6, 12.783-12.793. 